\section{Autoimmune Hemolytic Anemia Induced by Adalimumab}

Key words: adalimumab, anti-tumor necrosis factor agents, autoimmune hemolytic anemia, rheumatoid arthritis, psoriasis

\section{(Intern Med 55: 715, 2016)}

(DOI: 10.2169/internalmedicine.55.5773)

To the Editor We read with great interest the recent article by Harada et al. titled "Autoimmune Hemolytic Anemia During Adalimumab Treatment for Plaque Psoriasis", which reported that autoimmune hemolytic anemia (AIHA) might be induced in a patient with psoriasis by long-term adalimumab treatment (1). However, we disagree with their conclusion.

Their patient developed AIHA after treatment with adalimumab for 3 years (1), however, a 3-year exposure period is quite unusual for drug-induced adverse events. Antitumor necrosis factor (TNF) agents are well known to induce autoimmune phenomena, including vasculitis, systemic lupus erythematosus (SLE), and psoriasis. The mean duration of exposure was reported to be 38 weeks for vasculitis and 41 weeks for SLE (2), and the median exposure time was reportedly 6 months for psoriasis (3). Hematological complications of anti-TNF agents are rare (4). With regard to thrombocytopenia and neutropenia associated with antiTNF agents, the reported exposure time has ranged from 1 to 67 weeks and 2 to 56 weeks, respectively (4). Harada et al. cited three reports concerning the induction of AIHA by monoclonal antibodies (1). In two of the three reports, there is at least chronological plausibility, since AIHA was induced after 6 and 8 months of treatment. The remaining article reported an experimental study on the toxicity of an anti-HM1.24 monoclonal antibody; AIHA was induced after one month of repeatedly administering a toxic dose of this antibody to monkeys.

Harada et al. suggested that the mechanism of hemolytic anemia was related to the formation of complexes between adalimumab and "anti-adalimumab antibody" on erythro- cytes, which stimulate the immune system to produce antierythrocyte antibodies. However, this mechanism was derived from the above-mentioned animal toxicity study of anti-HM1.24 antibody and it may not apply to the present patient.

Drug-induced immune hemolytic anemia is very rare (5). It has mainly been reported with antibiotics, followed by nonsteroidal anti-inflammatory drugs, antineoplastic agents, and antihypertensives/diuretics. Hemolytic anemia induced by cephalosporins occurs soon after the start of treatment, with a mean duration until onset of 9 and 12 days for cefotetan- and ceftriaxone-induced hemolytic anemia, respectively. Garratty suggested that if patients with AIHA have anti-erythrocyte antibodies, then idiopathic warm-type AIHA should be primarily considered because it is much more common than drug-induced immune hemolytic anemia and the drug history may be a "red herring" (5).

While it is important for clinicians to report uncommon drug-induced adverse events, the causality must be assessed carefully.

The authors state that they have no Conflict of Interest (COI).

Takao Nagashima and Seiji Minota

\section{References}

1. Harada Y, Yamamoto H, Sato M, Kodaira M, Kono T. Autoimmune hemolytic anemia during adalimumab treatment for plaque psoriasis. Intern Med 54: 1103-1104, 2015.

2. Ramos-Casals M, Brito-Zeron P, Munoz S, et al. Autoimmune diseases induced by TNF-targeted therapies: analysis of 233 cases. Medicine (Baltimore) 86: 242-251, 2007.

3. Harrison MJ, Dixon WG, Watson KD, et al. Rates of new-onset psoriasis in patients with rheumatoid arthritis receiving antitumour necrosis factor alpha therapy: results from the British Society for Rheumatology Biologics Register. Ann Rheum Dis 68: 209-215, 2009.

4. Bessissow T, Renard M, Hoffman I, Vermeire S, Rutgeerts P, Van Assche G. Review article: non-malignant haematological complications of anti-tumour necrosis factor alpha therapy. Aliment Pharmacol Ther 36: 312-323, 2012.

5. Garratty G. Immune hemolytic anemia associated with drug therapy. Blood Rev 24: 143-150, 2010.

Division of Rheumatology and Clinical Immunology, Department of Medicine, Jichi Medical University, Japan

Received for publication May 14, 2015; Accepted for publication June 16, 2015

Correspondence to Dr. Takao Nagashima, naga4ma@jichi.ac.jp

(C) 2016 The Japanese Society of Internal Medicine Journal Website: http://www.naika.or.jp/imonline/index.html 